UCRL-JC-126901 Rev 2

PREPRINT

\title{
Femtosecond Laser Materials Processing
}

\author{
B. C. Stuart \\ P. S. Banks \\ M. D. Perry
}

This paper was prepared for submittal to the

Manufacturing '98

Chicago, IL

September 9-16, 1998

August 5, 1998

This is a preprint of a paper intended for publication in a journal or proceedings. Since changes may be made before publication, this preprint is made available with the understanding that it will not be cited or reproduced without the permission of the author. 


\section{DISCLAIMER}

This document was prepared as an account of work sponsored by an agency of the United States Government. Neither the United States Government nor the University of California nor any of their employees, makes any warranty, express or implied, or assumes any legal liability or responsibility for the accuracy, completeness, or usefulness of any information, apparatus, product, or process disclosed, or represents that its use would not infringe privately owned rights. Reference herein to any specific commercial product, process, or service by trade name, trademark, manufacturer, or otherwise, does not necessarily constitute or imply its endorsement, recommendation, or favoring by the United States Government or the University of California. The views and opinions of authors expressed herein do not necessarily state or reflect those of the United States Government or the University of California, and shall not be used for advertising or product endorsement purposes. 


\title{
Femtosecond Laser Materials Processing
}

\author{
B. C. Stuart, P. S. Banks, M. D. Perry \\ Lawrence Livermore National Laboratory \\ P.O. Box 808, L-477, Livermore, CA 94550 \\ (925) 424-5782, stuart3@llnl.gov
}

\begin{abstract}
Femtosecond lasers enable materials processing of most any material with extremely high precision and negligible shock or thermal loading to the surrounding area. Applications ranging from drilling teeth to cutting explosives to precision cuts in composites are possible by using this technology. For material removal at reasonable rates, we have developed a fully computer-controlled 15-Watt average power, 100-fs laser machining system.
\end{abstract}

\section{INTRODUCTION}

The use of femtosecond lasers allows materials processing of practically any material with extremely high precision and minimal collateral damage. Advantages over conventional laser machining (using pulses longer than a few tens of picoseconds) are realized by depositing the laser energy into the electrons of the material on a time scale short compared to the transfer time of this energy to the bulk of the material (either electron-phonon coupling or thermal diffusion), resulting in increased ablation efficiency and negligible shock or thermal stress. We present several examples here which demonstrate the benefits of, and in many cases are enabled by, using the technology of femtosecond laser materials processing.

The physical nature of the short-pulse laser interaction with material (along with the benefits) results in only a very thin layer $(\approx 0.1-1 \mu \mathrm{m})$ of material being ablated by each pulse. For efficient bulk material removal, laser systems of high repetition rate and high average power are necessary. In addition, for femtosecond laser materials processing to gain industrial acceptance, the laser systems must be made turn-key and reliable. We have taken a step in that direction by constructing a computer controlled, 15-W average power system that requires no laser knowledge or adjustments on the part of the operator.

\section{DIELECTRICS}

In transparent dielectric materials ablated with high-intensity femtosecond pulses, very little energy (shock or thermal) is coupled into the bulk material because the pulse duration is shorter than the characteristic time for energy transfer from the electrons to the lattice (a few ps for most dielectrics). Measured and calculated ablation thresholds for fused silica ${ }^{1}$ are shown in Figure 1 over a wide range of pulse duration. The deviation from long-pulse behavior ( $\tau^{1 / 2}$ scaling) at $\approx 20$ ps signals the transition into 
this new regime of nonthermal ablation. The continuously decreasing threshold for shorter pulses means that less energy is coupled into surrounding areas as shock or thermal loading.

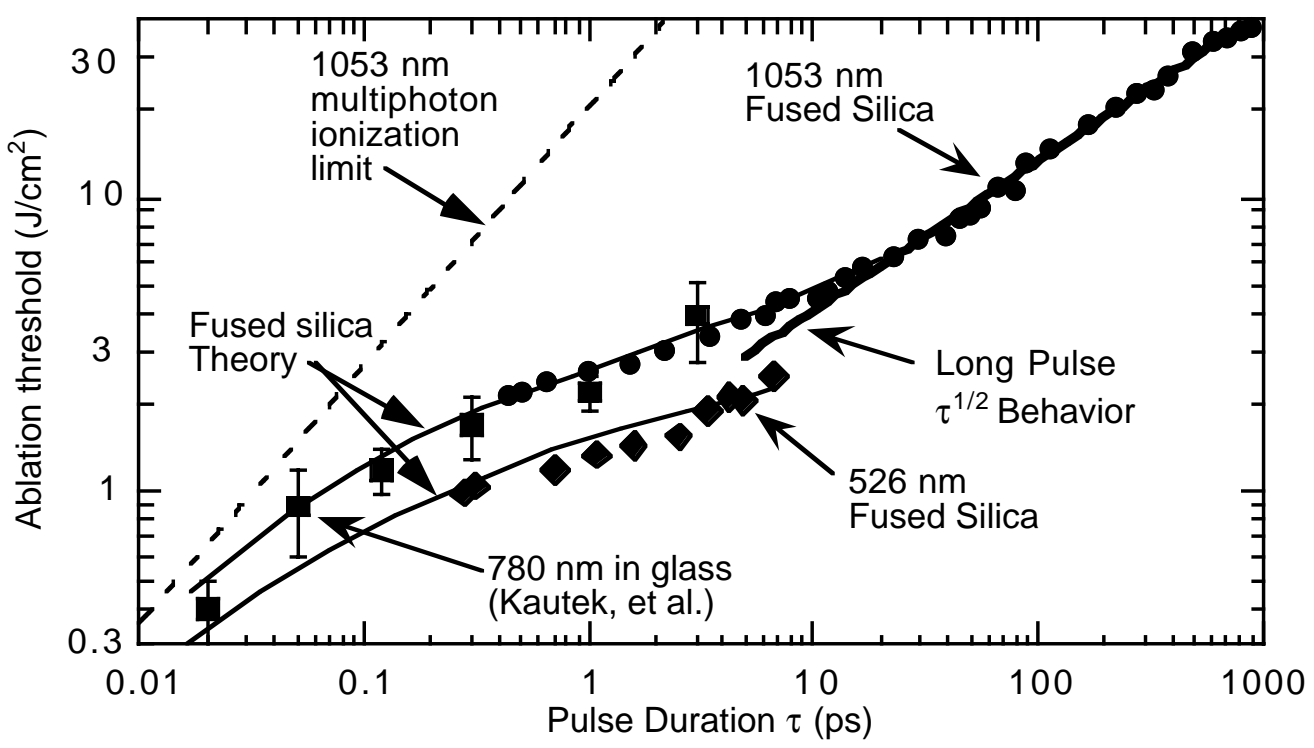

Figure 1. Measured (¥ fused silica $1053 \mathrm{~nm}$; fused silica $526 \mathrm{~nm} ; 4$ glass ${ }^{2} 780 \mathrm{~nm}$ ) and calculated (solid line) ablation threshold. Deviation from $\tau^{1 / 2}$ scaling below 20 ps indicates the new regime of nonthermal ablation.

From the standpoint of this mechanism of laser interaction, enamel, bone, etc. are simply dielectrics and exhibit the same behavior as fused silica. Shown in Figure 2 are electron micrographs of the surface of a tooth following material removal by 1.4-ns laser pulses and 350-fs pulses. The pulses are produced by a variable pulse duration laser operating at $1053 \mathrm{~nm}$ and $10 \mathrm{~Hz}$. Cracking of the surrounding material and the uncontrollable nature of material removal by thermal shock are readily apparent in the enamel irradiated by nanosecond pulses (Fig. 2a). In this regime, linear absorption due to defects produces inhomogeneous energy absorption across the laser beam and thermal stresses build up causing ablation first from the point with the least material strength. This is not the case with femtosecond pulses where all regions throughout the laser beam profile with sufficient intensity to be above ablation threshold will be removed resulting in extremely fine control of the position of material removal (Fig. 2b). In addition, the morphology in the femtosecond case is characteristic of internal enamel and there is no evidence of heat transfer into the surrounding material.

This non-thermal material removal mechanism results in a minimal increase in temperature of the surrounding material. Thermal measurements show that when irradiated with 500 conventional 1-ns laser pulses, the bulk temperature of a 1-mm slice of tooth increased by over $40{ }^{\circ} \mathrm{C}$ while for femtosecond pulses the temperature rise was less than $2^{\circ} \mathrm{C}$ (Figure 3). The fluence in each case was set to remove approximately $1 \mu \mathrm{m}$ depth of material per pulse. This required $30 \mathrm{~J} / \mathrm{cm}^{2}$ for the ns pulses and only $3 \mathrm{~J} / \mathrm{cm}^{2}$ for the fs pulses. The practical consequences in dentistry are substantial. In the case of existing laser systems, active cooling of the tooth is necessary to prevent permanent damage to the pulp $\left(5^{\circ} \mathrm{C}\right.$ increase) while no cooling would be necessary with femtosecond laser pulses. 


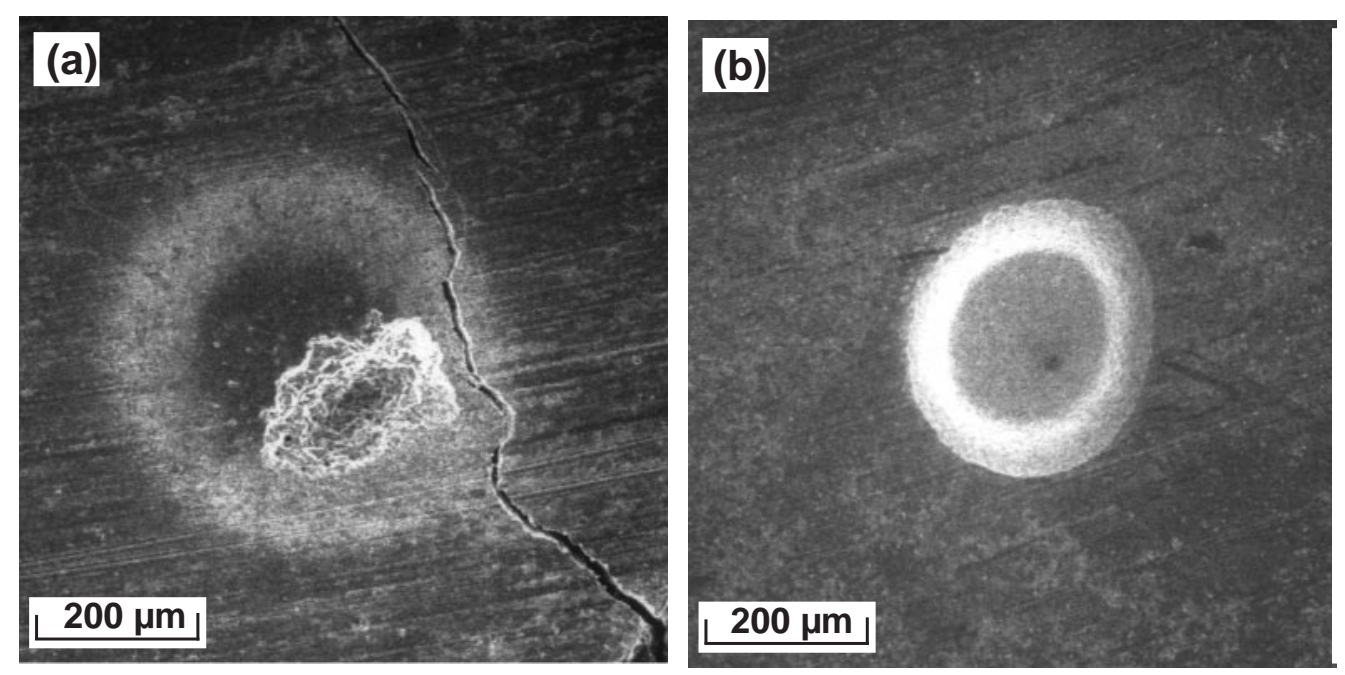

Figure 2. (a) Drilling of enamel with $1.4 \mathrm{~ns}, 30 \mathrm{~J} / \mathrm{cm}^{2}$ laser pulses. Enamel is removed by a conventional thermal/fracture mechanism resulting in cracking and collateral damage from temperature rise and thermal shock. (b) Drilling of enamel with $350 \mathrm{fs}, 3 \mathrm{~J} / \mathrm{cm}^{2}$ laser pulses. Enamel is removed at the same rate by a nonthermal mechanism which eliminates collateral damage and leaves the surface in its natural state.

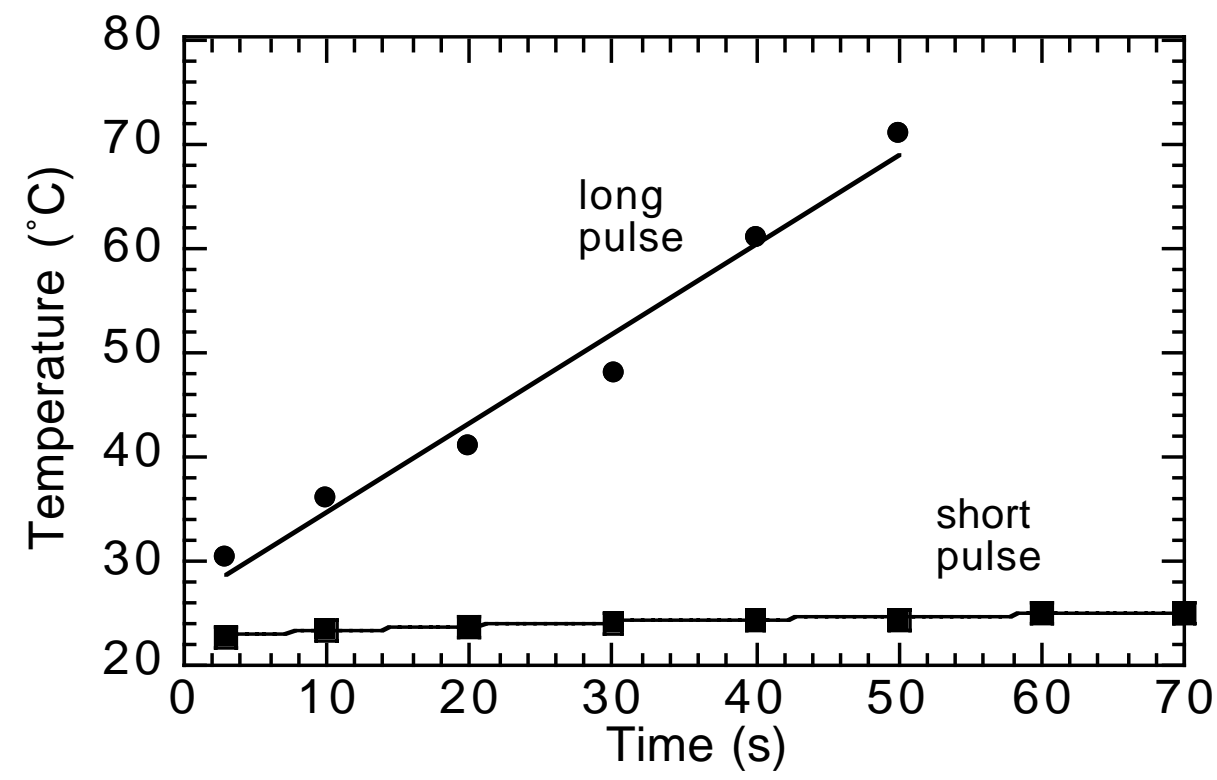

Figure 3. Temperature increase of bulk tooth due to drilling with nanosecond (circles) and femtosecond (squares) laser pulses. In both cases, the laser wavelength was $1053 \mathrm{~nm}$ and the material removal rate was 1 $\mu \mathrm{m} /$ pulse at $10 \mathrm{~Hz}$.

Another class of dielectric materials that greatly benefit from the reduced thermal and shock loading during femtosecond laser machining is that of high explosives (HE). Cutting and machining operations on energetic materials present significant safety challenges. If conventional machine tools are used, improper fixturing of the work, improper tool configuration and improper cutting speeds have resulted in violent reactions during machining operations. In addition, significant hazardous waste is generated from the machining chips and the necessity of using cutting fluids to cool the cutting tool. 
Ablation of high explosives by femtosecond laser pulses offers an attractive alternative to conventional machining. Absorption of these ultra-short pulses occurs on such a short time scale that the material is ablated with virtually no heat transfer to the surrounding material, resulting in a "cold" laser cutting process.

We used LX-16 explosive (96\% PETN) for our initial experiments because PETN is one of the most sensitive of the secondary explosives. In some of the experiments the beam first cut through the HE pellet and then into a stainless steel substrate and in other experiments the beam first cut through stainless steel and then into the HE pellet. In either case, no reaction was observed. We also cut through pellets that were not backed by a substrate. Figure 4a shows two cuts across a 6-mm diameter, 2-mm thick pellet made using a $1-\mathrm{kHz}, 100$-fs laser system. The ablation process is very efficient in explosives with several microns of material being removed per pulse. We also observed for small spot sizes (approximately 25 $\mu \mathrm{m})$ that after an initial taper, the cut exhibits extremely straight walls extending for several millimeters. This enables cuts with extremely high aspect ratios of 200:1 and more.

The only experiment in which reaction was observed was in cutting a LX-16 pellet when we did not compress the pulse after amplification (a factor of 5000 increase in pulse length). Using 500-ps pulses at a power level high enough to remove material, ignition of the LX-16 was observed. Examination of the pellet afterward revealed that the edges of the cut were melted and contained a multitude of reaction products (Fig. 4b).
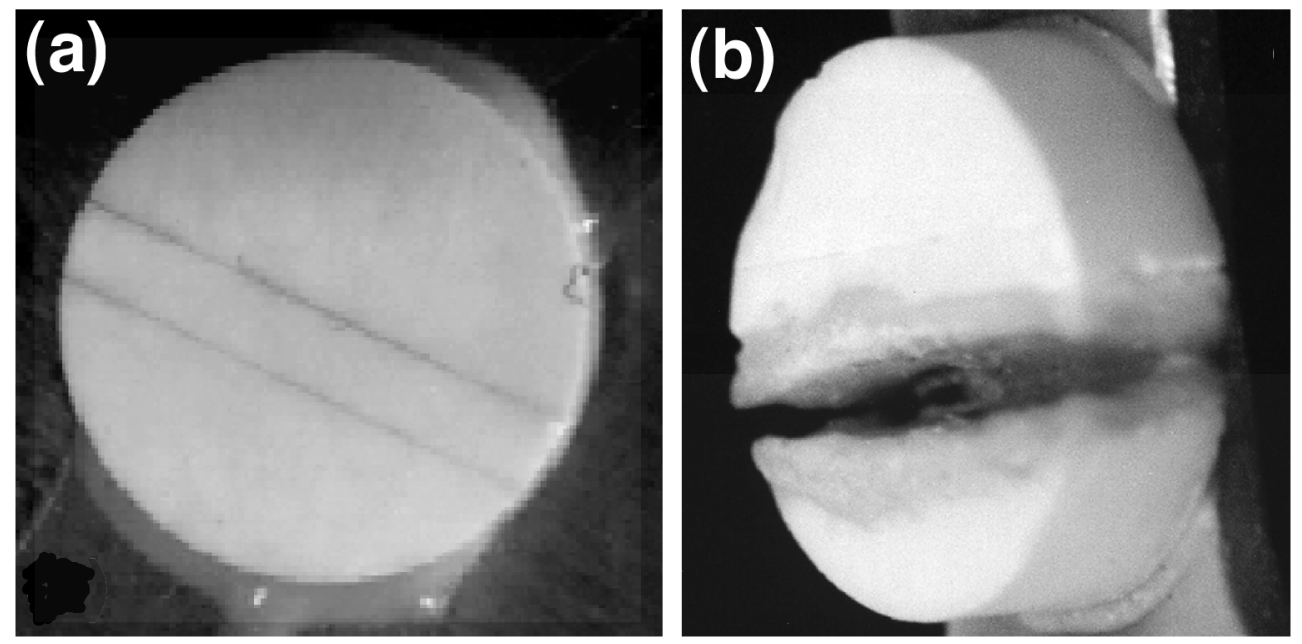

Figure 4. Cuts in explosive pellet (PETN) by (a) short pulse and (b) long pulse laser. Thermal deposition in the long-pulse case caused the pellet to ignite.

Preliminary investigations show that the benefits of localized ablation can be realized in nontransparent and composite dielectric materials as well. Figure 5 shows a $300-\mu \mathrm{m}$ diameter hole and a 300$\mu \mathrm{m}$ wide line cut in two different carbon-epoxy samples. Very precise cuts with clean edges are made possible by this technology. 

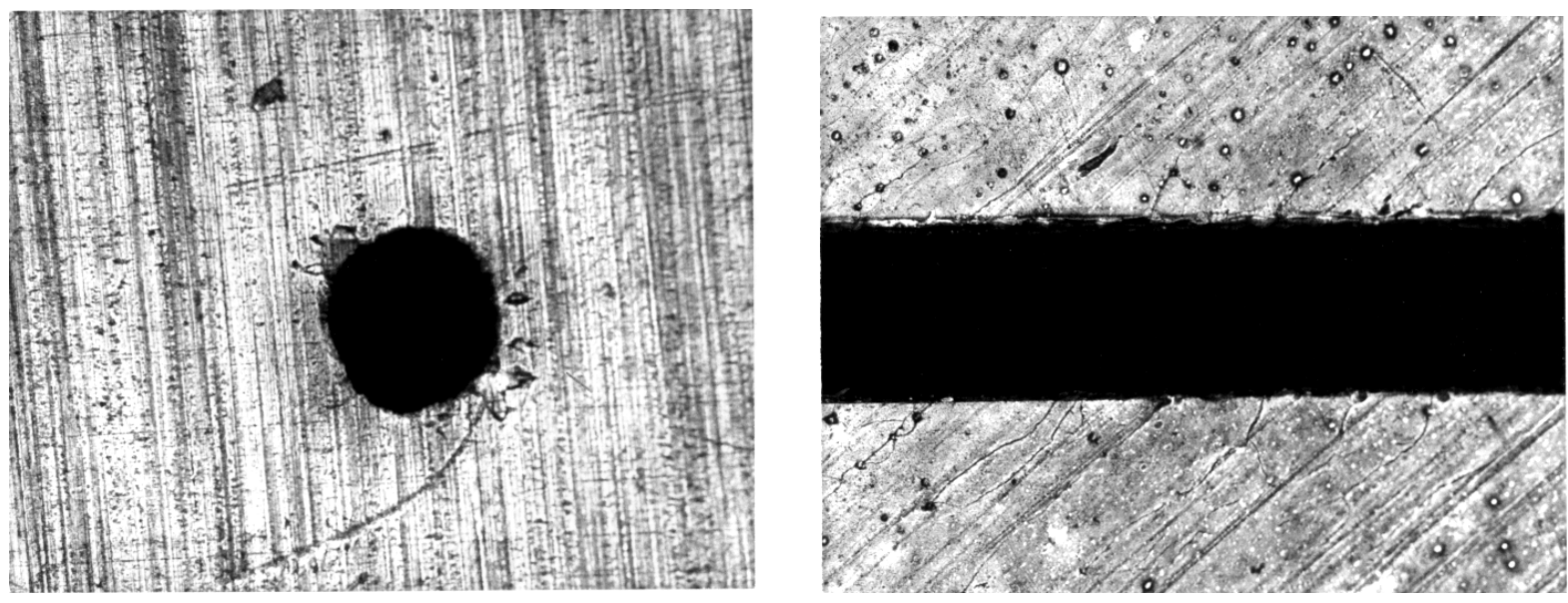

Figure 5. Hole and line cut in carbon epoxy with femtosecond laser pulses.

\section{METALS}

In metals, by choosing the laser pulse duration such that the thermal diffusion depth is on the order of the distance the laser light penetrates, very small amounts of material $(0.01-1 \mu \mathrm{m})$ can be precisely removed with minimal transport of energy by either shock or thermal conduction away from the volume of interest The vaporized material expands from the surface with little energy deposited in the bulk. Thus, there is no heat-affected zone in the remaining bulk material nor recast layer. Figure 6 shows the crosssection of a $400-\mu \mathrm{m}$ hole in $750 \mu \mathrm{m}$ thick stainless steel at $45^{\circ}$; the metallic grain structure is unaltered up to the edge of the cut. The top (entrance) of this cut is shown in Fig. 7, again with no evidence of slag or heataffected zone.
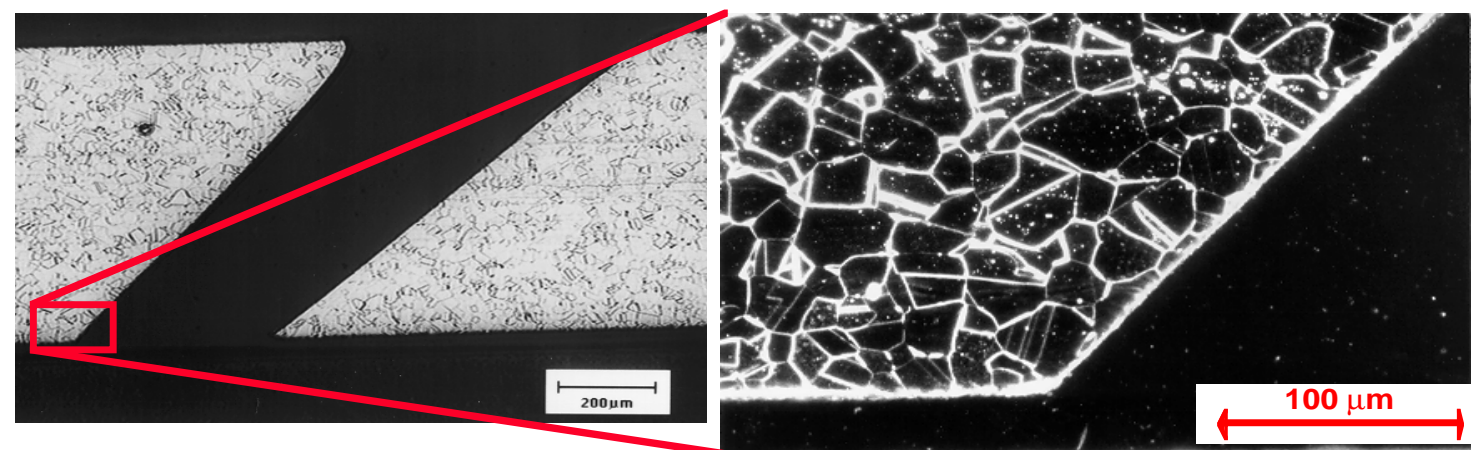

Figure 6. Cross section of hole drilled in stainless steel with 120 -fs pulses at $1 \mathrm{kHz}$. 


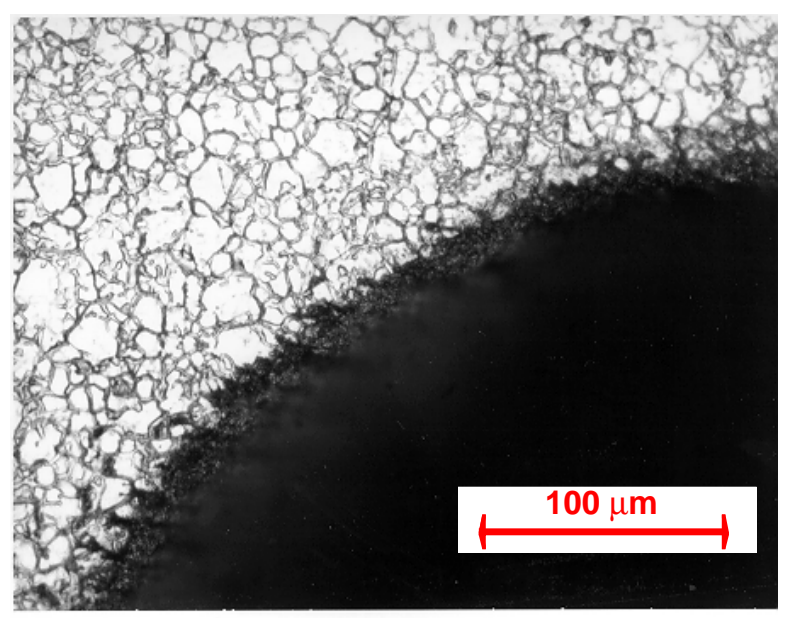

Figure 7. Top of hole drilled in stainless steel. There is no evidence of melting or slag.

\section{15-W SHORT-PULSE LASER PROCESSING SYSTEM}

The rate of material removal scal es essentially linearly with the average power of the laser system. We have recently completed a high average power short-pulse laser system for use in an industrial environment. The system is fully computer-controlled and is designed to be run by a machine-tool operator. A block diagram of the system is shown in Fig. 8. It is based on conventional technology, i.e. arclamp pumped, intracavity-doubled Nd:YAG pumping Ti:sapphire, and was designed to operate without the added complexity of cryogenic cooling of the Ti:sapphire crystals.

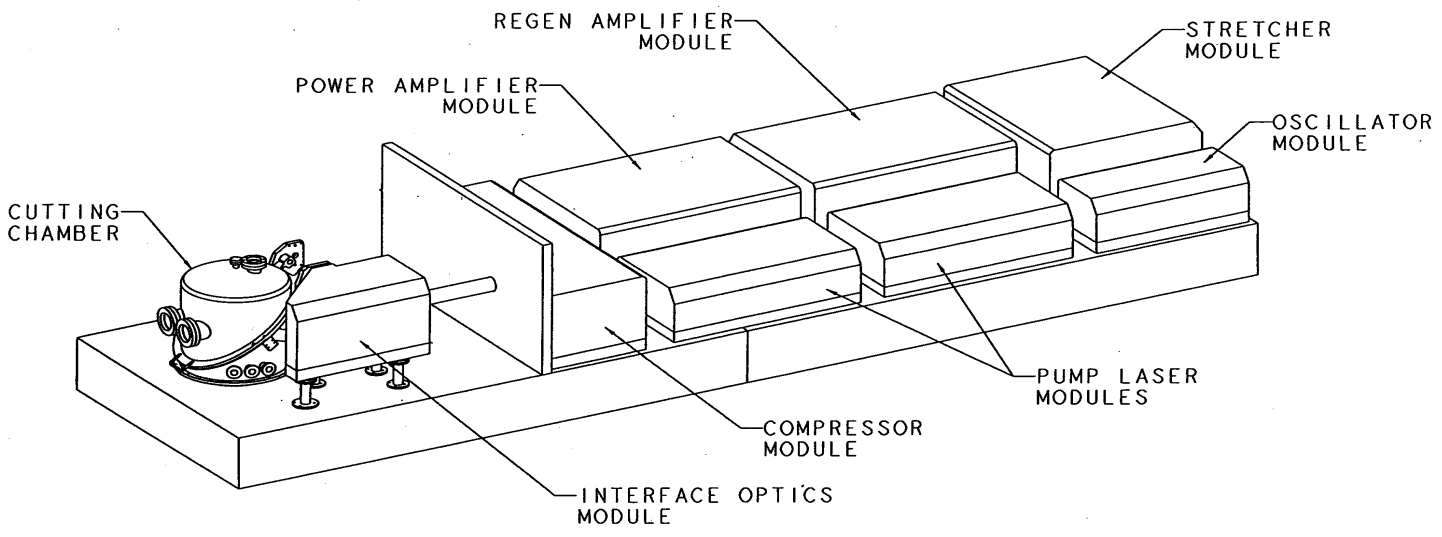

Figure 8. Configuration of $15-\mathrm{W}$ short-pulse machining system.

This work was performed under the auspices of the U.S. Department of Energy by Lawrence Livermore National Laboratory under contract No. W-7405-ENG-48.

${ }^{1}$ B.C. Stuart, M.D. Feit, A.M. Rubenchik, B.W. Shore, and M.D. Perry, "Laser-induced damage in dielectrics with nanosecond to subpicosecond pulses", Phys. Rev. Lett. 74, pp. 2248-2251, 1995.

${ }^{2}$ W. Kautek, J. Kruger, M. Lenzner, S. Sartania, C. Spielmann, F. Krausz, "Laser ablation of dielectrics with pulse durations between 20 fs and 3 ps", Appl. Phys. Lett. 69, pp. 3146-3148, 1996. 